\title{
Comparison between the Proactive, Reactive and Hybrid Routing Protocols
}

\author{
Sandeep Singh Rana ${ }^{1}$, Sunita Malik ${ }^{2}$ \\ M.Tech Scholar, ECE Dept, DCRUST Murthal ${ }^{1}$ \\ Asst. Prof, ECE Dept, DCRUST Murthal ${ }^{2}$
}

\begin{abstract}
Ad- hoc network is the network comprised of centralized base network and a mobilized network which communicate with each other. While communicating with each other, sensor nodes determine the shortest path to the destination nodes. As the sensor node operates over the limited battery power, it is very important to determine the efficient algorithm and routing protocols to fulfill the energy requirements of the network. To make the network secure, faster and energy efficient, we have to make changes in the routing protocols at the various (MAC and PHY) layer of the networks. In this paper, we carried out these changes to the LANMAR, DSR AND ZRP protocols and studied the effects in term of Throughput, end to end delay and Jitter at the application layer. The simulation results are carried out in QualNet 6.1 Simulator.
\end{abstract}

Keywords: Routing Protocols, Constant Bits Rate, Hop Count Etc.

\section{INTRODUCTION}

Ad-hoc Network is one of the widely used wireless network comprised of individual devices use to communicate to each other directly. In order to communicate with each other, sensor nodes first determine the routing path [1] from source to the destination nodes. Then the shortest path algorithm is selected to deliver the packets to the destination node. The different routing protocols have the different method of determining the routing path and each routing protocols has its own advantages and disadvantage. Hence the selection of routing protocols is purely based on the network or scenario we want to create. For example, to make the system faster and secure, we have to compromise with the efficiency or vice-versa.

\section{ROUTING PROTOCOLS}

Routing Protocols can be classified into: Proactive, Reactive and Hybrid Routing protocols. Proactive routing protocols are the routing protocols which are based on table driven or in which routing path are updated periodically by the sensor nodes whereas Reactive Routing protocols are the routing protocols in which routing path are maintained on demand of the network. On the other hand, Hybrid routing protocols are the routing protocols which combined the features of both the proactive and reactive protocols. In hybrid routing protocols, complete network is divided into zones. Within each zones the routing path is based on table driven method and outside the zone routing path is based on demand routing method. Selection of routing protocols [2] is entirely based on application scenario and our requirements. We prefer the routing protocols which suited our design standards and made the adjustment changes to the properties of the routing protocols.

\section{A. Dynamic Source Routing (DSR)}

DSR is On Demand or reactive routing protocols in which it accumulates the address of the source and destination node during route discovery. DSR consist of two phase: Route Discovery and Route Maintenance. When the source node wants to send packets to the destination node, it first sends the route request to the near nodes which consist of source address, destination address, node request ID. It will check where the destination node is and route reply to the source node about the destination address. In route maintenance phase, if the node discovery fails, it will send the route error to the source node and request for route reply message and update its cache memory.

\section{B. Landmark Routing Protocols (LANMAR)}

Landmark routing protocols combine the features of fisheye protocols and landmark routing. In lanmar, each node maintains the local routes to the landmark nodes. This information is used to determine the destination address .Based on the routing table, If the node is present within the scope packets are send directly to the destination node otherwise packets are send to the nearest landmark nodes. The benefits of LANMAR routing protocols is dramatically reduction of both table size and routing overhead, which is helpful in scalable it to large networks. 


\section{UGC Approved Journal}

\section{Zone based routing protocols (ZRP)}

Zone based routing protocols is the hybrid protocols which [4] combination the features proactive and reactive protocols. It takes the advantages of proactive discovery of within the local zones radius and using the reactive routing for communicating between the neighbor zone radius. In a limited zone radius, the routing information can be maintained easily and the amount of routing information that is never used is also minimized. The size of zone radius is defined by the number of hop counts. Zone routing protocols includes many components such as: Intra-zone routing protocols (IARP), Inter-zone routing protocols (IERP) and Bordercast routing protocols (BRP) which provides the full advantages to the ZRP protocols.

\section{Stimulation Setup}

In this paper, QualNet 6.1 stimulator is used to evaluate the performance of routing protocols. We have changed the properties of routing protocols to enhance the performance and observe the effects caused due to these changes. In DSR 1 and DSR 2 ,

Table 1: Simulation parameters values

\begin{tabular}{|c|c|c|}
\hline Simulation Parameters & \multicolumn{2}{|c|}{ Values } \\
\hline No. of nodes & \multicolumn{2}{|c|}{$10,40,80,100$} \\
\hline Dimension of space & \multicolumn{2}{|c|}{$1500 * 1500$} \\
\hline Radio types & $802.11 \mathrm{~b}$ & Multiple Access with Collision Avoidance (MACA) \\
\hline Simulation time & \multicolumn{2}{|l|}{$300 \mathrm{sec}$} \\
\hline Constant Bit rate(CBR) & \multicolumn{2}{|c|}{$\begin{array}{l}\text { For } 10 \text { node- }\{(1-3),(1-2)\} \\
\text { For } 40 \text { node }-\{(14-26),(13-26)\} \\
\text { For } 80 \text { node }-\{(56-97),(16-42)\} \\
\text { For } 100 \text { node }-\{(41-46),(23-30)\}\end{array}$} \\
\hline Mobility & \multicolumn{2}{|c|}{ None } \\
\hline Energy Model & \multicolumn{2}{|c|}{ Mica-Motes } \\
\hline Battery Model & \multicolumn{2}{|c|}{ Linear Model } \\
\hline Simulator & \multicolumn{2}{|c|}{ Qualnet 6.1 } \\
\hline Total Simulation & \multicolumn{2}{|c|}{15} \\
\hline
\end{tabular}

We have changed the MAC layer protocols: 802.11 and Medium access collision avoidance (MACA) resp. In LANMAR 1 and LANMAR 2, we have change the alpha factor and fisheye hop count from 1.3 and 2 to 1.5 and 4 respectively. In ZRP1 and ZRP 2, we have change the Zone radius and IERP buffer size from 2 and 100 to 4 and 256 respectively.

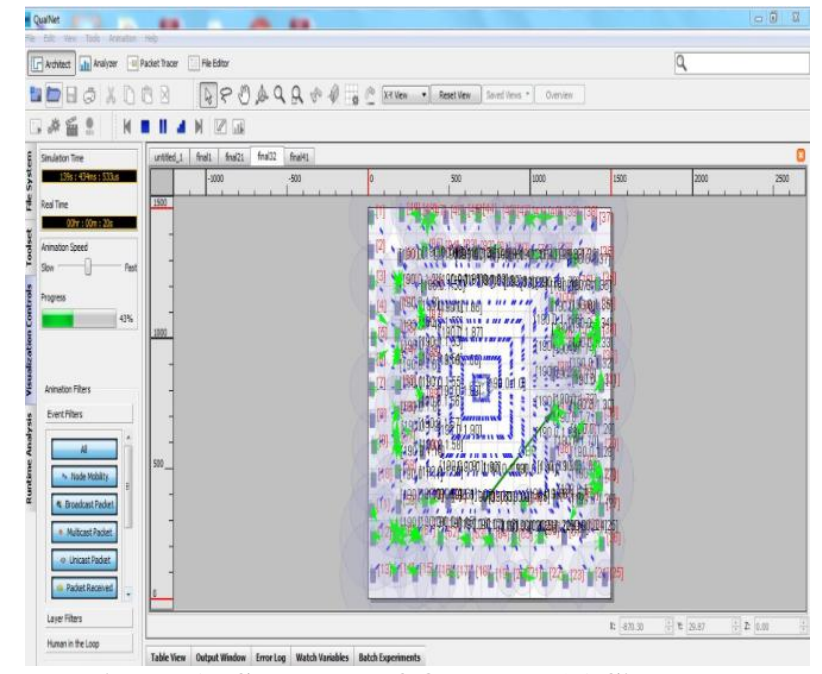

Figure 1 Snapshot of QualNet 6.1 Simulator

The constant bit rate is provided between the source and destination nodes. The constant bit rate applied for ZRP form source to destination nodes are from $\{(1-2),(13-26),(16-42),(23-30)\}$ and for LANMAR and DSR constant bit rate is between $\{(1-3),(14-26),(56-97)$ and (41-26) $\}$. The simulation is carried out for different network densities of 10, 40, 80, and 100 respectively. 
UGC Approved Journal

\section{A. End to end delay:}

It indicates how much time is taken to send packets from the constant bits rate source to the destination nodes in the application layer. In DSR, ZRP and LANMAR, with the increase in a number of nodes, the mean end to end delay decreases comparatively. The mean end to end delay of DSR, LANMAR AND ZRP is shown below in the figure 2, figure 3 and figure 4 respectively.

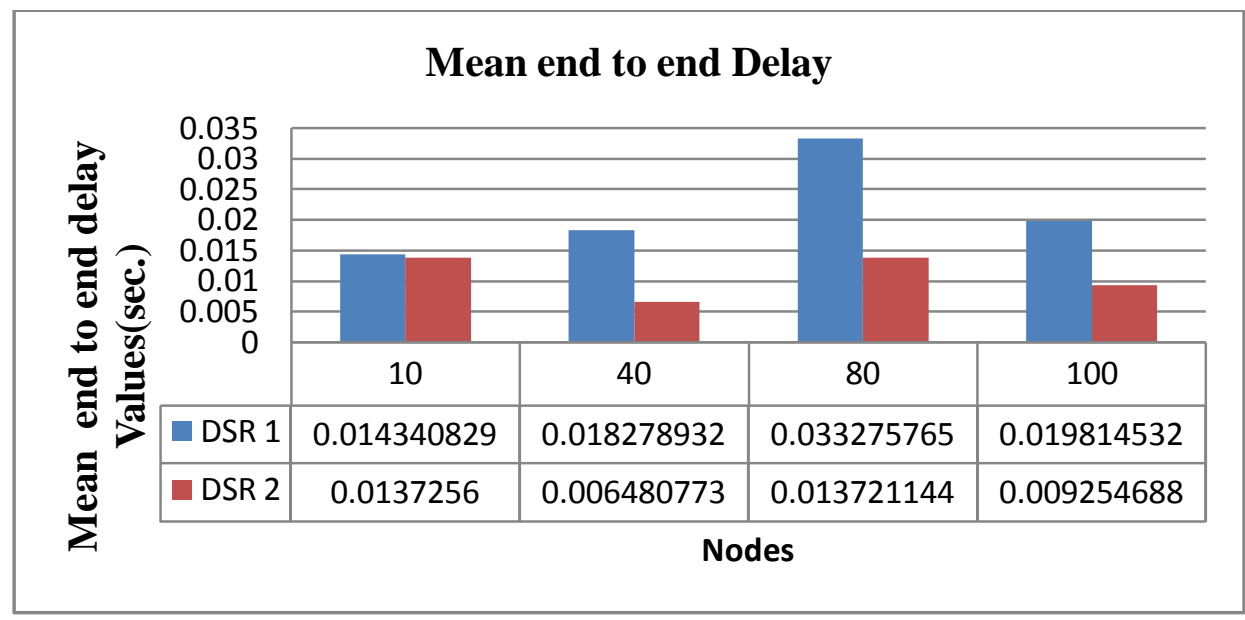

Figure 2 Mean end to end delay of DSR protocols.

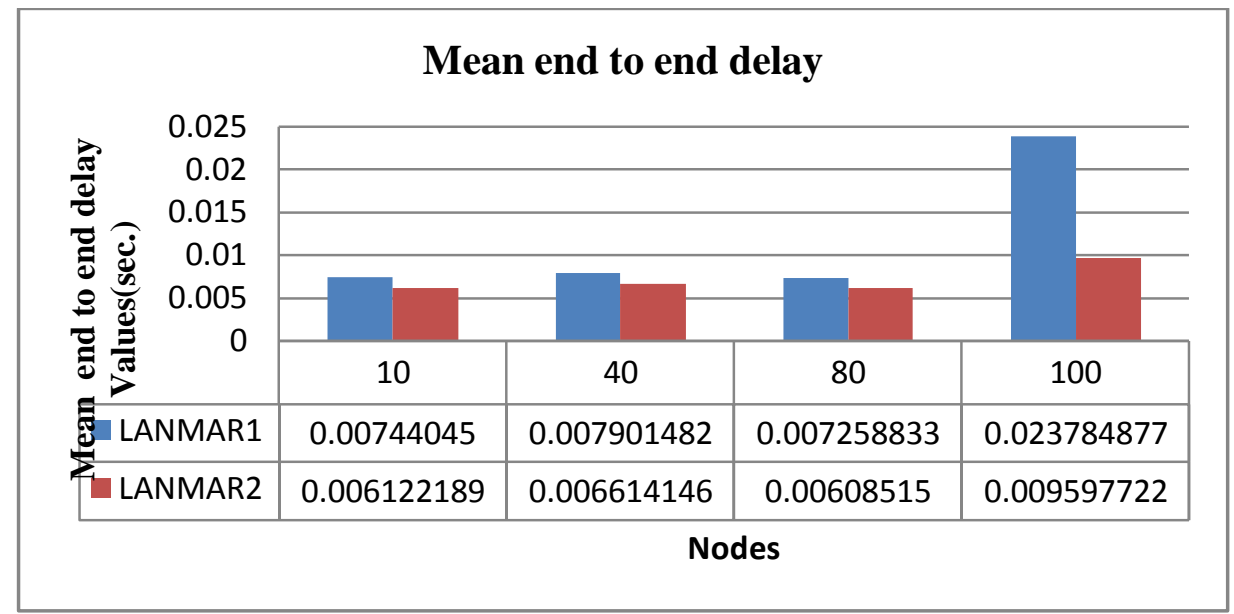

Figure 3 Mean end to end delay of LANMAR protocols.

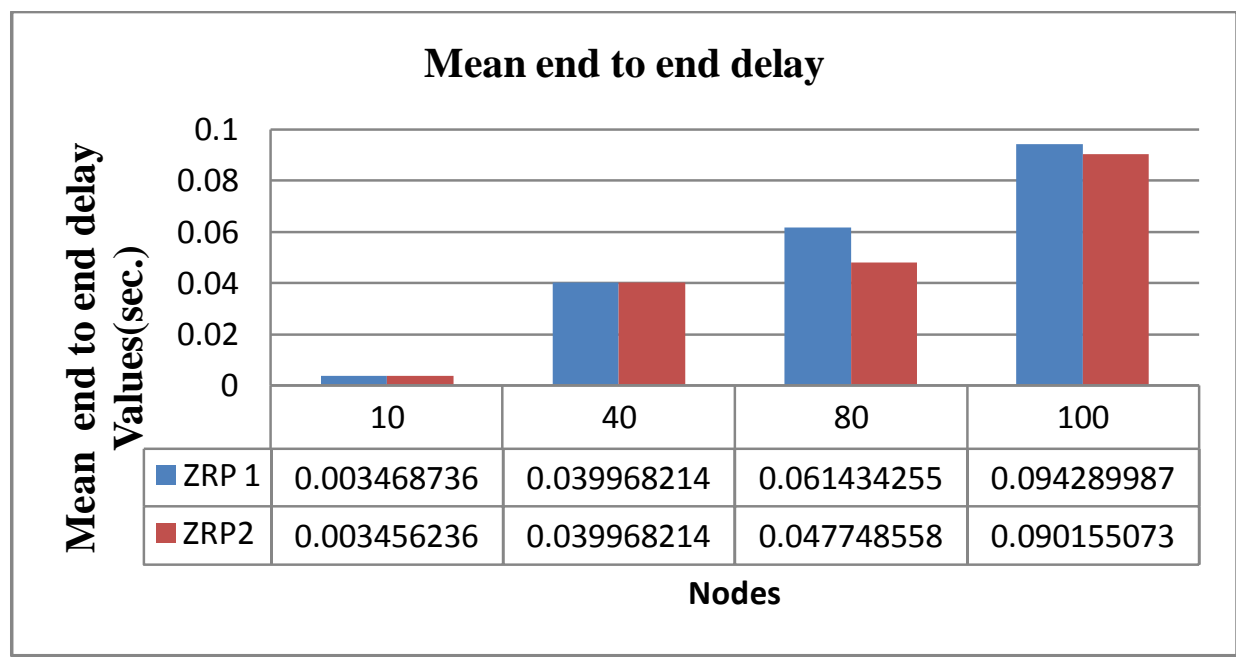

Figure 4 Mean end to end delay of ZRP protocols 


\section{B. Throughput :}

It is defined as the total data received at the receiver node from the sender divided by the time taken to receive the last packets. It is measured in bits per second (bits per second). In DSR and LANMAR, with an increase in the number of nodes, throughput decreases. However in 40 nodes scenario, it decreases largely and in case of 80 nodes scenario, the decrease is very small. In ZRP, Throughput is lest affected and are nearly same. The Throughput at the application layer of DSR, LANMAR AND ZRP is shown below in the figure 5, figure 6 and figure 7 respectively.

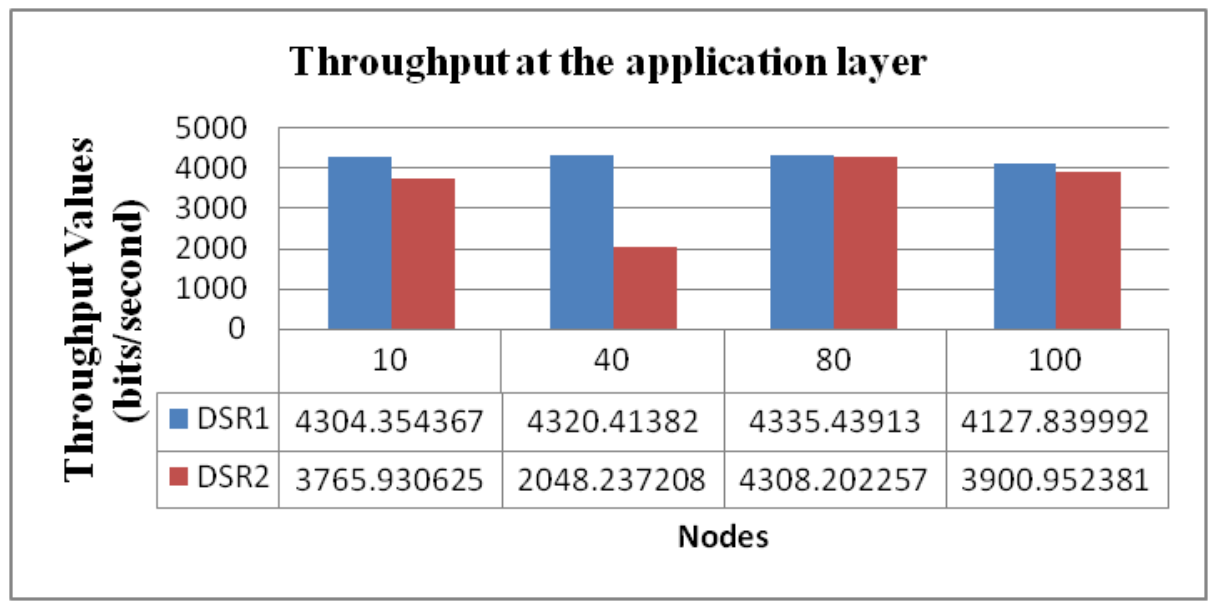

Figure 5 Throughput at the application layer of DSR protocols.

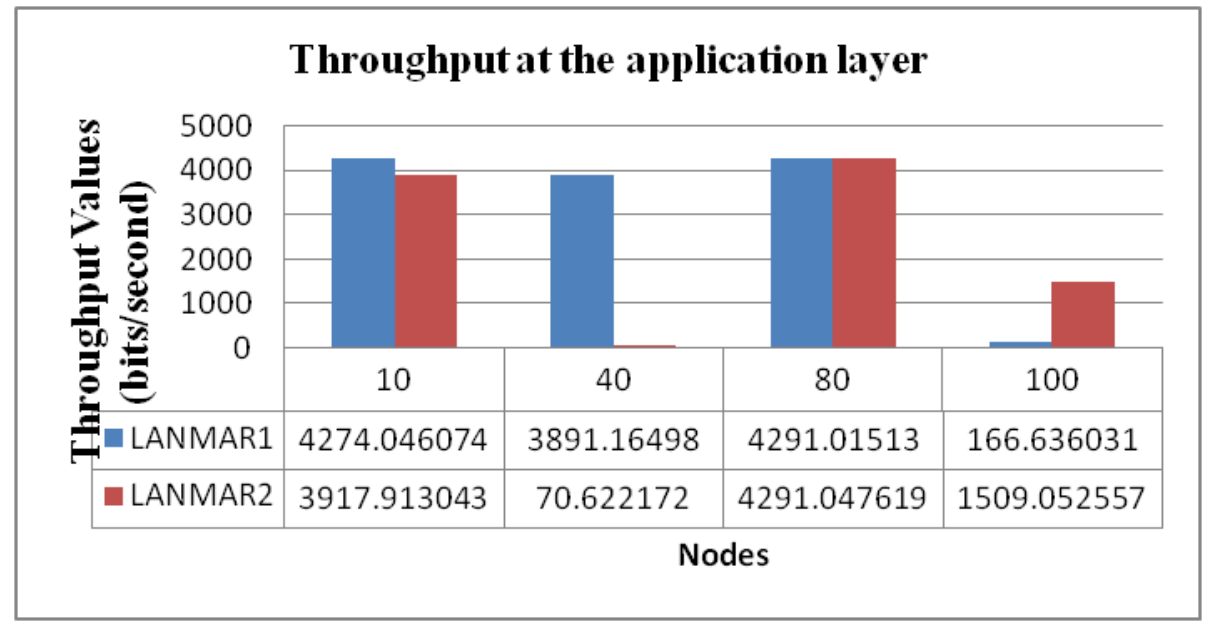

Figure 6 Throughput at the application layer of LANMAR protocols.

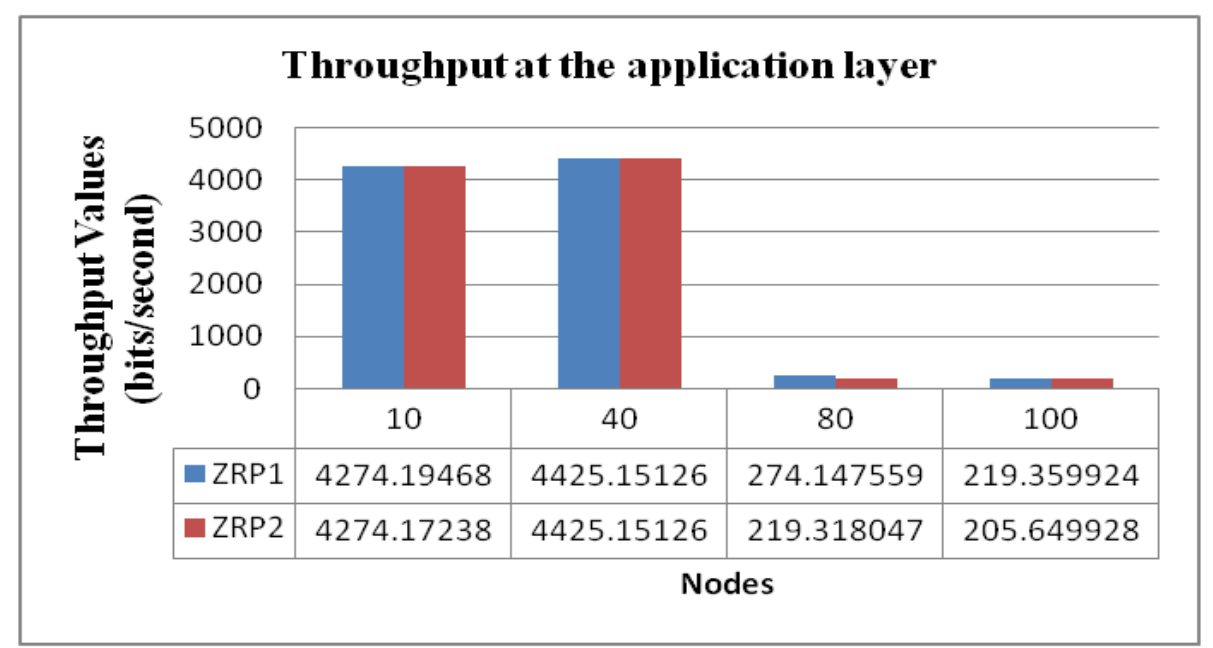

Figure 7 Throughput at the application layer of ZRP protocols. 


\section{Average Jitter:}

Jitter is defined as the variation in the time interval between the packets received at the destination nodes caused by the network congestion, route changes or timing drifting. Jitter must be as small as possible for the better performance of the network. In DSR and LANMAR, with the increase in a number of nodes, Jitter decrease comparatively large. Whereas in ZRP, with the increase in the number of nodes fluctuation in the jitter is least. The Jitter at the application layer of DSR, LANMAR AND ZRP is shown below in the figure 8, figure 9 and figure 10 respectively.

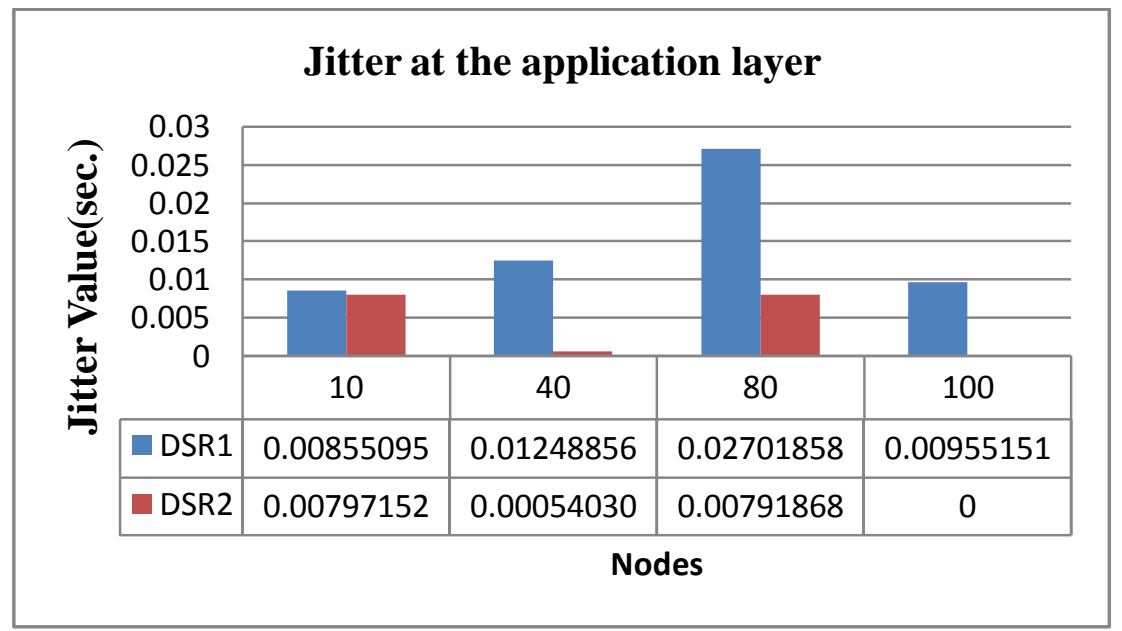

Figure 8 Jitter at the application layer of DSR protocols.

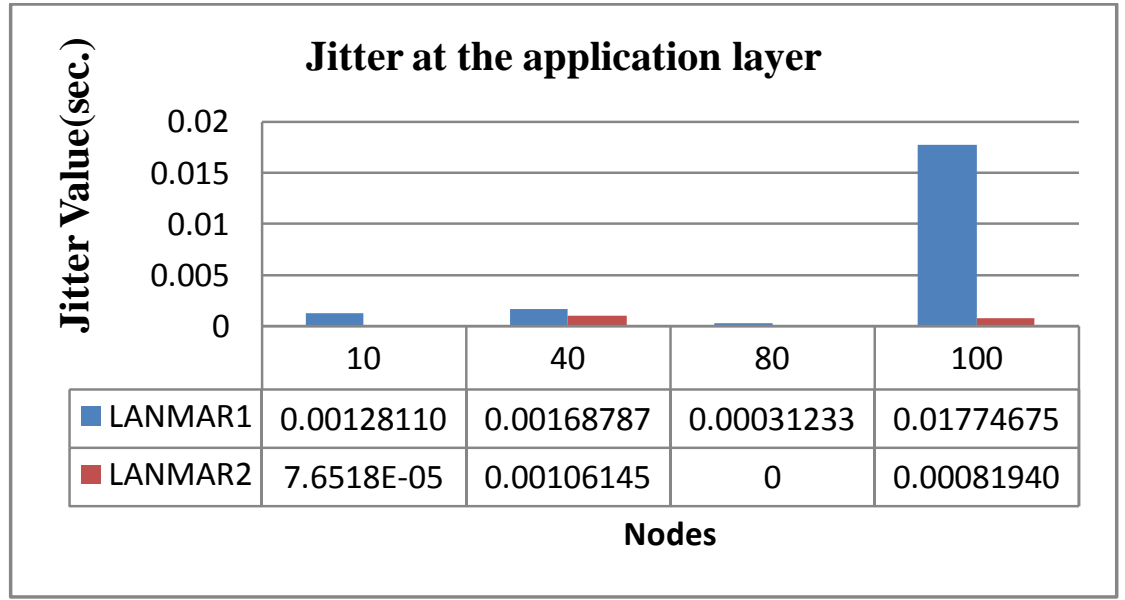

Figure 9 Jitter at the application layer of LANMAR protocols.

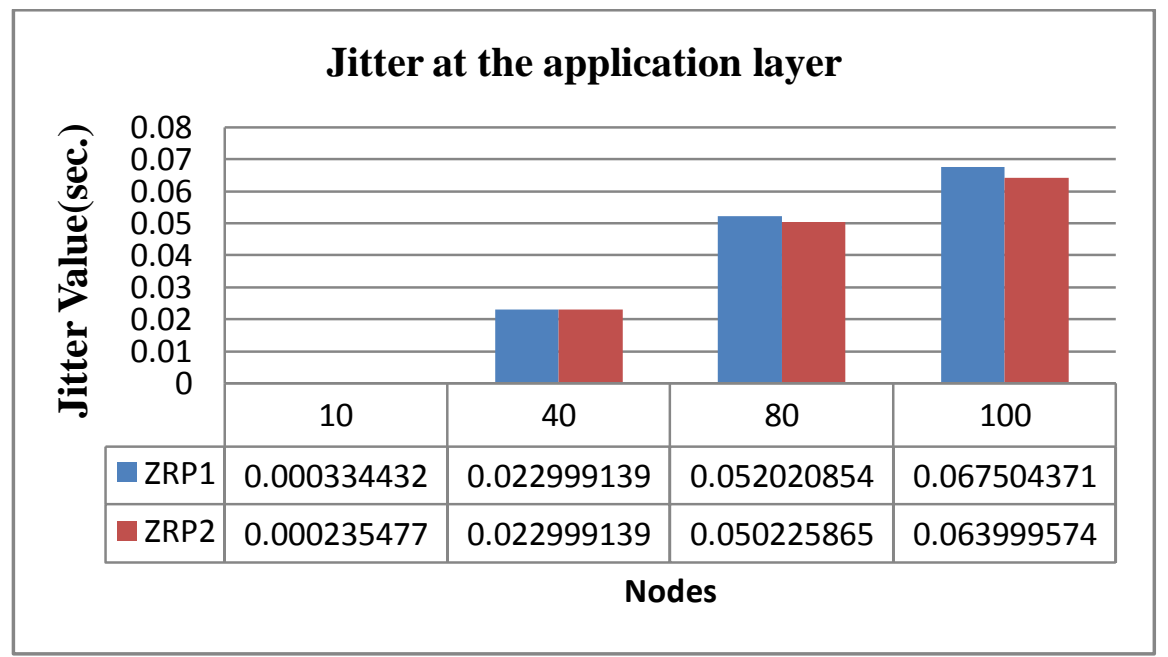

Figure 10 Jitter at the application layer of ZRP protocols. 


\section{CONCLUSION}

In this paper, the performance of different routing protocols such as DSR, LANMAR and ZRP is evaluated under the effect of varying the properties of these routing protocols. It is evaluated from the above experiment that all the three performance evaluating factors are depending on each other. When there is end to end delay in the packet transmission increases from the source node to destination, it causes a probability of losing the packets. Similarly Jitter also affects the performance of the network. The decrease in jitter causes a less probability of dropping the packets and congesting in the network. Throughout of the network is the most affected factor with increase or decrease in network end to end delay and jitter. In case of ZRP1 and ZRP2, throughput is almost equal to each other. There are many other factors which affect the throughput to decrease such as: placement of nodes, mobility, network congestion to the destination nodes etc. All The evaluation of performance is carried out in the QualNet 6.1 simulator. We can further extend this work, by evaluating the effects of various battery models, network layer protocols, antenna patterns and MAC layer protocols on the different routing protocols.

\section{REFERENCES}

[1]. Kemal Akkaya and Mohamed Younis, “A Survey on Routing Protocols for Wireless Sensor Networks”, Ad hoc Networks, vol. 3, no. 3, May 2005 .

[2]. Lee Kok Thong (2004) "Performance analysis of mobile adhoc routing protocols" thesis Naval post graduate college, Monterey, California.

[3]. Anne Aaron, Jie Weng, "Performance Comparison of Ad-hoc Routing Protocols for Networks with Node Energy Constraints", available at http://ivms.stanford.edu, Spring 2000-2001.

[4]. Z.J. Hass, R. Pearlman, (1999) Zone routing protocol for ad-hoc networks, Internet Draft, draft ietf-manet-zrp-02.txt

[5]. R.K. Chauhan and Ashish Chopra, "Power Optimization In Mobile Ad Hoc Network", Global Journal of Computer Science and Technology, Vol. 10 Issue 4 Ver. 1.0 June 2010, pp 92-96.

[6]. Sandeep Singh Rana, Sunita Malik, "Evaluation of Qualnet of Service of Different Routing Protocols using QualNet 6.1 Simulator", Volume 8, No. 5, May - June 2017,International Journal of Advanced Research in Computer Science (IJARCS), ISSN No.0976-5697,pp- 2007-2010.

[7]. Qualnet Simulator www.scalable-networks.com 